\title{
B7 homolog 6 promotes the progression of cervical cancer
}

\author{
RUIMENG GUO ${ }^{1}$, GUOYAN LIU ${ }^{2}$, CHANGYING LI $^{3}$, XUEJING LIU ${ }^{4}$, \\ YANYING XU ${ }^{4}$, WEINA YANG ${ }^{4}$ and FANG WANG ${ }^{4}$ \\ ${ }^{1}$ Department of Gynecology, The Second Hospital of Tianjin Medical University, Tianjin 300211; \\ ${ }^{2}$ Department of Gynecology, The General Hospital of Tianjin Medical University, Tianjin 300053; \\ ${ }^{3}$ Tianjin Institute of Urology and ${ }^{4}$ Department of Gynecology, The Second Hospital \\ of Tianjin Medical University, Tianjin 300211, P.R. China
}

Received September 15, 2020; Accepted April 12, 2021

DOI: $10.3892 / \mathrm{etm} .2021 .10206$

\begin{abstract}
B7 homolog 6 (B7-H6) was recently discovered to act as a co-stimulatory molecule. In particular, the expression of B7-H6 has been found to play an important biological role in several types of tumors. The aim of the present study was to determine the role of B7-H6 in cervical cancer. Immunohistochemistry was used to analyze the expression levels of B7-H6 in cervical precancerous and cancerous tissues. Furthermore, the expression of B7-H6 was knocked down in HeLa cells using short hairpin RNA and the effects of B7-H6 on HeLa cell proliferation, migration and invasion were determined using Cell Counting Kit-8, colony formation, wound healing and Transwell invasion assays, respectively. In addition, flow cytometry was used to analyze the levels of cell apoptosis and the cell cycle distribution. The results of the immunohistochemical staining revealed that the expression levels of B7-H6 were upregulated in cervical lesions. Furthermore, the expression levels of B7-H6 were positively associated with the clinical stage of the cervical lesions. B7-H6 knockdown suppressed the invasive, migratory and proliferative abilities of HeLa cells, and promoted G1 cell cycle arrest and apoptosis. In conclusion, the findings of the present study suggested that B7-H6 may serve as a novel oncogene and may hold promise as a potential therapeutic target for cervical cancer.
\end{abstract}

\section{Introduction}

Cervical cancer is the fourth most common type of cancer diagnosed in women worldwide, accounting for $>500,000$ new cases and 311,365 deaths in 2018 (1). Despite cervical cancer being one of the more treatable malignant diseases, with

Correspondence to: Dr Guoyan Liu, Department of Gynecology, The General Hospital of Tianjin Medical University, 154 Anshan Road, Tianjin 300053, P.R. China

E-mail: liuguoyan211@126.com

Key words: B7 homolog 6, cervical cancer, HeLa cells, biological function, apoptosis, cell cycle multiple treatment strategies available, including surgical resection, radiation and chemotherapy, there are currently no effective targeted or immunotherapeutic approaches for patients with advanced cervical cancer $(2,3)$. Therefore, it is important to identify additional molecules that could provide novel treatment strategies for cervical cancer.

B7 homolog 6 (B7-H6), which is a member of the B7 family, has been attracting increasing attention from researchers due to its reported role in tumorigenesis $(4,5)$. B7-H6, as a ligand of NKp30, which is an activating receptor of natural killer (NK) cells (6-8), was found to play an important role in tumor immune surveillance and escape (9-14). Notably, B7-H6 expression is rarely reported in normal tissues and is mainly expressed in numerous types of cancer, including esophageal squamous cell carcinoma, oral squamous cell carcinoma and breast cancer (4). In recent years, B7-H6 expression has been reported to be upregulated in certain cancers and to be associated with tumor differentiation, stage and progression. For example, Zhou et al (15) retrospectively analyzed the clinical data of 145 patients with esophageal squamous cell carcinoma and found that B7-H6 expression levels were upregulated in esophageal cancer tissues, and were associated with tumor size, stage and lymph node metastasis. Sun et al (16) also used immunohistochemistry to investigate the expression of B7-H6 in 305 patients with breast cancer. The results revealed that the expression levels of B7-H6 in breast cancer tissues were positively associated with tumor progression. Furthermore, Wang et al (17) used immunohistochemistry to analyze the expression of B7-H6 in 50 patients with oral squamous cell carcinoma, and found that the expression levels of B7-H6 in oral squamous cell carcinoma tissues were significantly upregulated compared with those in the normal oral mucosa, and were significantly associated with cancer differentiation. However, to the best of our knowledge, the clinical significance and role of B7-H6 in cervical cancer have not been investigated to date.

To explore whether B7-H6 may be a potential therapeutic target for cervical cancer, the present study used immunohistochemical analysis to determine the association between B7-H6 expression and the physiology of cervical cancer. Furthermore, short hairpin RNA (shRNA), cell function experiments, apoptosis and cell cycle analysis were used to determine the possible role of B7-H6 in the regulation of the biological behavior of cervical cancer. 


\section{Materials and methods}

Patients and samples. Archived cervical pathological tissue samples from the Department of Pathology of Second Hospital of Tianjin Medical University (Tianjin, China) obtained between November 2015 and August 2019 were used in the present study. Each disease stage comprised 30 samples. The age range of patients with cervical intraepithelial neoplasia (CIN) grade I (CIN I), CIN II, CIN III and cervical cancer was 44-56, 42-57, 43-53 and 44-64 years, respectively, with a median age of $50 \pm 8.11,49 \pm 10.23,47 \pm 6.02$ and $54 \pm 9.42$ years, respectively. Furthermore, 30 normal cervical samples (median patient age, $53 \pm 2.53$ years; range, $49-58$ years) were included in the present study. There were no statistically significant differences in age among the groups $(\mathrm{P}=0.773)$. The research protocol was approved (approval no. KY2019K023) by the Ethics Committee of The Second Hospital of Tianjin Medical University and the patients or their family members provided written informed consent for their tissues to be used for research purposes prior to participation.

The following inclusion criteria were used: i) Normal cervical tissue samples were obtained from patients undergoing hysterectomy with negative ThinPrep cytology test (TCT) and human papillomavirus results; ii) CIN I and CIN II tissues were obtained from patients who had undergone a cervical biopsy; iii) CIN III (18) tissues were obtained from patients who had undergone cervical conization; and iv) cervical cancer tissues were obtained from patients who had undergone a cervical diagnostic biopsy or therapeutic surgery. All specimens were examined and the diagnosis was confirmed by pathological analysis, and the TCTs revealed that $<30 \%$ cervial inflammatory cells were found in each sample. The following exclusion criteria were used: i) Body mass index $\geq 25 \mathrm{~kg} / \mathrm{m}^{2}$; ii) levels of biochemical markers (such as serum lipid profile, creatinine and liver function tests) not within the normal range; iii) smokers; and iv) presence of other systemic diseases.

Immunohistochemistry. The cervical pathological tissue from the patients recruited at our hospital were fixed with $10 \%$ formaldehyde for $24 \mathrm{~h}$ at room temperature and embedded in paraffin. Every array block was cut into $4-\mu \mathrm{m}$ sections, deparaffinized with xylene and rehydrated in a descending series of alcohol. Antigen retrieval was performed by heating the tissue sections in sodium citrate buffer $(0.01 \mathrm{mmol} / \mathrm{l}$; $\mathrm{pH} 6.0$ ) at $100^{\circ} \mathrm{C}$ for $30 \mathrm{~min}$. Cooled sections were subsequently immersed in $3 \%$ hydrogen peroxide solution at $37^{\circ} \mathrm{C}$ for $10 \mathrm{~min}$ to block the endogenous peroxidase activity and then incubated with an anti-B7-H6 rabbit antibody (1:150; cat. no. ab121794; Abcam) at $4^{\circ} \mathrm{C}$ overnight. Following incubation with the primary antibody, the sections were incubated with an HRP-conjugated goat anti-rabbit secondary antibody (1:100; cat. no. abs975; Absin Bioscience, Inc.) at room temperature for $1 \mathrm{~h}$. The sections were subsequently counterstained with hematoxylin for $30 \mathrm{sec}$ at room temperature and $\mathrm{DAB}$ for 1 min was used for chromogen detection at room temperature. Finally, the sections were dehydrated using an ascending alcohol series, cleared and sealed with neutral resin. Samples incubated with PBS instead of the primary antibody served as negative controls (NC).
Evaluation of immunohistochemical staining. Using the positive control cells as reference, the immunostaining intensity of B7-H6, which stained the cytoplasm and cell membrane, was assessed using the $\mathrm{H}$-score. Notably, only $3 \%$ interstitial cells were weakly stained with B7-H6, and these interstitial cells were associated with the inflammatory reaction that occurred around the lesion. In the process of counting, only positive cells in the cervical epithelium were considered, while the positive cells in the interstitium were not counted. The H-score was calculated using the following equation: $\%$ of unstained cells $\mathrm{x}(0+\%$ of weakly stained cells $) \times(1+\%$ of moderately stained cells) $\mathrm{x}(2+\%$ of strongly stained cells) $\mathrm{x} 3(10)$. A total of three high-density areas were determined for each specimen under a low-power microscope (magnification, x100), and each high-density area was subsequently counted under a high-power light microscope (magnification, x200); the mean value of the three areas was obtained.

Cell culture. The HeLa cervical cancer cell line was gifted by Dr Xue Du (Department of Gynecology, The General Hospital of Tianjin Medical University, Tianjin, China). HeLa cells were cultured in RPMI-1640 medium (cat. no. 31870074; Invitrogen; Thermo Fisher Scientific, Inc.) supplemented with 10\% FBS (cat. no. F8318; Sigma-Aldrich; Merck KGaA), and maintained at $37^{\circ} \mathrm{C}$ in a humidified atmosphere containing $5 \% \mathrm{CO}_{2}$.

Cell transfection. Two shRNAs of $28 \mu \mathrm{g} / \mathrm{ml}$ targeting B7-H6 (shRNA-1,5'-CCCTGCTCTCCTAACAGTT-3'; and shRNA-2, 5'-GGTTCTACCCAGAGGCTAT-3') and shRNA-NC (5'-TTC TCCGAACGTGTCACGT-3') were synthesized by Suzhou GenePharma Co., Ltd. (cat. nos. 190331CZ, 190331DZ and C03DZ, respectively). The pGLV3/H1/GFP (LV) lentiviral vectors LV3-shRNA-1, LV3-shRNA-2 or LV3-shRNA-NC (MOI, 10) were transfected into HeLa cells at room temperature, and screened with polybrene $(1.5 \mu \mathrm{g} / \mathrm{ml}, 1: 1,000$; Suzhou GenePharma Co., Ltd.). Viral infections were performed serially. The fluorescence microscope was used to observe whether GFP fluorescence transfection efficiency was $>80 \%$ after $48 \mathrm{~h}$ of transfection. Stable cell lines expressing shRNAs were selected with $2 \mu \mathrm{g} / \mathrm{ml}$ puromycin $72 \mathrm{~h}$ after transfection.

Reverse transcription-quantitative PCR (RT-qPCR) analysis. RT-qPCR was used to detect the B7-H6 mRNA level in HeLa cells transfected with the three different shRNAs. Total RNA was extracted from cultured cells using TRIzol ${ }^{\circledR}$ reagent (cat. no. 213407; Invitrogen; Thermo Fisher Scientific, Inc.) according to the manufacturer's instructions. Purified RNA was then reversely transcribed to cDNA using RevertAid First Strand cDNA Synthesis kit purchased from Thermo Fisher Scientific, Inc. Reverse transcription was performed at $42^{\circ} \mathrm{C}$ for $1 \mathrm{~h}$ and the reaction was terminated by heating at $70^{\circ} \mathrm{C}$ for $5 \mathrm{~min}$. Next, qPCR was performed with FastStart Universal SYBR-Green Master Mix (cat. no. 04913850001; Roche Diagnostics) following the manufacturer's instructions. An initial amplification using B7-H6 specific primers was performed with a denaturation step at $95^{\circ} \mathrm{C}$ for $10 \mathrm{~min}$, followed by 35 cycles of denaturation at $95^{\circ} \mathrm{C}$ for $60 \mathrm{sec}$, primer annealing at $58^{\circ} \mathrm{C}$ for $30 \mathrm{sec}$ and primer extension at $72^{\circ} \mathrm{C}$ for $30 \mathrm{sec}$. Data were normalized to the geometric mean of the housekeeping gene GAPDH to control 
the variability in expression levels and calculated as $2^{-\Delta \Delta \mathrm{Cq}}$ method (19). The primer sequences of B7-H6 were as follows: Forward 5'-TTTTCCATTCATTGGTGGCCTA-3' and reverse 5'-TTTTCCATTCATTGGTGGCCTA-3'. The primer sequences of GAPDH were as follows: Forward 5'-CTGGAACGGTGA AGGTGACA-3' and reverse 5'-AAGGGACTTCCTGTAACA ATGCA-3'.

Western blotting. HeLa cells were lysed with RIPA lysis buffer (cat. no. BL504A; Biosharp; http:/www.biosharp. cn/index/product/details/language/en/product_id/1783.html), and the protein concentration was determined using the Quick-Start Bradford protein assay (Bio-Rad Laboratories, Inc.). Equal amounts of protein (30 $\mu \mathrm{g}$ loaded per lane) were separated via $10 \%$ SDS-PAGE and transferred onto PVDF membranes (cat. no. P2938-1ROL; Sigma-Aldrich; Merck $\mathrm{KGaA}$ ). The membranes were blocked at room temperature for $1 \mathrm{~h}$ with TBS- $0.05 \%$ Tween-20 containing 5\% skimmed milk powder. The membranes were subsequently incubated with an anti-B7-H6 rabbit antibody (1:1,000; cat. no. ab121794; Abcam) or an anti-GAPDH antibody (1:5,000; cat. no. G8795; Sigma-Aldrich; Merck KGaA) at $4^{\circ} \mathrm{C}$ overnight. Following primary antibody incubation, the membranes were incubated with a HRP-conjugated goat anti-rabbit (1:5,000; cat. no. abs20040; Absin Bioscience, Inc.) or goat anti-mouse secondary antibody (1:4,000; cat. no. abs20039; Absin Bioscience, Inc.) at room temperature for $1 \mathrm{~h}$. Protein bands were visualized using ECL reagent (cat. no. abs920; Absin Bioscience, Inc.). The ImageJ software (version no. v1.8.0; National Institutes of Health, Bethesda, MD) was used to quantify the WB data. The experiment was repeated three times.

Cell Counting Kit-8 (CCK-8) assay. Cell proliferation was determined using a CCK-8 assay (cat. no. 69092500; BioSharp Life Sciences). Briefly, the cells were digested by $1 \mathrm{ml}$ trypsin and seeded at a density of $2 \times 10^{3}$ cells/well into four 96 -well plates. Following 1,2, 3 and 4 days of incubation at room temperature, $10 \mu \mathrm{l} \mathrm{CCK}-8$ reagent was added/well and incubated for $4 \mathrm{~h}$ at room temperature. The optical density value of each well was measured at $450 \mathrm{~nm}$ using plate reader to generate the cell proliferation curves. The experiment was repeated three times.

Wound healing assay. The cells were digested, counted and seeded into a 6 -well plate at a density of $5 \times 10^{5}$ cells/well. When the cells reached $90 \%$ confluence, a linear scratch was created in the cell monolayer using a $10-\mu 1$ pipette tip. The non-adherent cells were removed by rinsing with $1 \mathrm{ml}$ PBS after creating the scratch. The cells were subsequently incubated in serum-free RPMI-1640 medium at $37^{\circ} \mathrm{C}$ for 0,24 or $48 \mathrm{~h}$. At the indicated time points, the plate was imaged with a light microscope (Olympus Corporation) at a magnification of x200 to observe the distance of cell migration, which was calculated using the following formula: Migration distance at $\mathrm{n} \mathrm{h}=$ (scratch width at $0 \mathrm{~h}$ - scratch width at $\mathrm{n} h$ ), where $\mathrm{n}$ represents each time point. The measurements were made at random intervals along the wound length. The experiment was repeated three times.

Transwell invasion assay. In total, $60 \mu 1$ Matrigel with $7 \mathrm{mg} / \mathrm{ml}$ (cat. no. 356237; Corning, Inc.) was evenly spread on the upper layer of the chamber at $37^{\circ} \mathrm{C}$ for $1 \mathrm{~h}$. The cells were digested with $1 \mathrm{ml}$ trypsin, counted and $2 \times 10^{4}$ cells were subsequently centrifuged at $161 \times \mathrm{g} 3 \mathrm{~min}$ at room temperature, followed by removal of the supernatant. The cells were resuspended in $100 \mu \mathrm{l}$ serum-free RPMI-1640 medium and plated into the upper chamber of 24-well Transwell plate, while $500 \mu \mathrm{l}$ RPMI-1640 medium supplemented with 10\% FBS was plated into the lower chamber. Following incubation for $48 \mathrm{~h}$ at $37^{\circ} \mathrm{C}$, the cells were fixed with $600 \mu \mathrm{l}$ paraformaldehyde (4\%) for $30 \mathrm{~min}$ at room temperature and stained at room temperature with $600 \mu \mathrm{l}$ crystal violet dye $(0.1 \%$; cat. no. G1061; Beijing Solarbio Science \& Technology Co., Ltd.) for $10 \mathrm{~min}$. The chambers were subsequently washed twice with PBS and dried. The number of invasive cells was counted in five randomly selected fields of view using an inverted fluorescent microscope (magnification, x100; Olympus Corporation). The experiment was repeated three times.

Colony formation assay. Cells at the logarithmic growth phase were collected when they reached a confluence of $90 \%$. After staining with $0.4 \%$ trypan blue dye for $3 \mathrm{~min}$ at room temperature, the number of living cells that were released into $1 \times 10^{3} / \mathrm{ml}$ suspension were counted. Complete culture medium $(1 \mathrm{ml})$ and cell suspension $(1 \mathrm{ml})$ was added into the 6-well plate $(3,000$ cells per well) successively, and the cells were dispersed by rotating slightly. The 6-well plate was placed in the incubator for further culture for 1 week at $37^{\circ} \mathrm{C}$ in a humidified atmosphere containing $5 \% \mathrm{CO}_{2}$. After removing the medium in the 6-well plate, the cells were washed twice with PBS and then fixed with $4 \%$ paraformaldehyde for $20 \mathrm{~min}$ at room temperature. After removing the paraformaldehyde and washing twice with PBS, $0.1 \%$ crystal violet dye was added to the 6 -well plate for $10 \mathrm{~min}$ at room temperature, then the excess dye was washed with PBS and the plate was dried with air. The transparent plate with a gridwas put on the bottom of the plate. The cell clone clusters containing $>50$ cells number in each hole was counted under a light microscope (Olympus Corporation) at a magnification of x100. Finally, the clone formation rate was calculated as follows: Clone formation rate $=$ (clone number/inoculated cell number $) \times 100 \%$. After analyzing the data of three independent repeat experiments, statistical analysis was carried out and a histogram was drawn.

Apoptosis and cell cycle analyses. Briefly, $1 \times 10^{6}$ cells from the shRNA-NC and shRNA-2 groups were washed with PBS. Apoptosis was detected using a PE-Annexin V apoptosis detection kit (cat. no. 559763; BD Biosciences). In total, $1 \times 10^{6}$ cells in a $100 \mu 1$ cell suspension was incubated with $5 \mu \mathrm{l}$ PE-Annexin $\mathrm{V}$ solution and $5 \mu \mathrm{l}$ propidium iodide (PI) solution (concentration, $10 \mu \mathrm{g} / \mathrm{ml}$ ) for $15 \mathrm{~min}$ in the dark at $4^{\circ} \mathrm{C}$. The experiment was repeated three times.

For cell cycle analysis, $1 \times 10^{6}$ cells in a $100 \mu \mathrm{l}$ cell suspension were stained with $0.5 \mathrm{ml} \mathrm{PI/RNase} \mathrm{staining} \mathrm{buffer}$ (cat. no. 550825; BD Biosciences) for $20 \mathrm{~min}$ in the dark at $4^{\circ} \mathrm{C}$. Finally, the stained cells of $1 \times 10^{6}$ in $100 \mu \mathrm{l}$ cell suspension were detected by the NovoCyte D3000 flow cytometer (cat. no. CA92121; ACEA Biosciences, Inc.) and the data were analyzed by the NovoExpress software (version no. 1.5; ACEA Biosciences, Inc.). The results in second (PI+ AV+; late apoptotic cells) and fourth (PI- AV+; early apoptotic cells) 

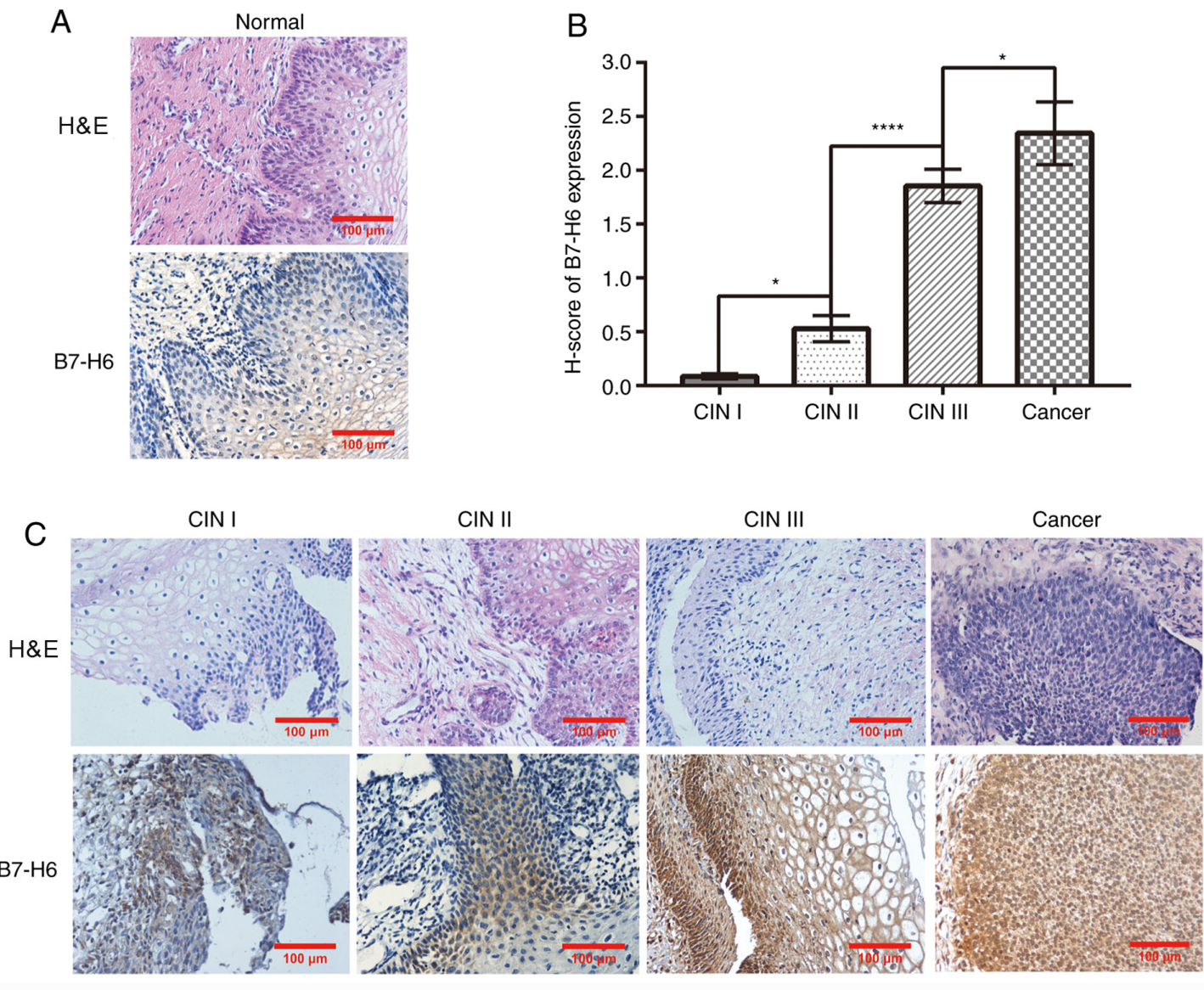

Figure 1. B7-H6 expression is associated with cervical cancer grade. (A) H\&E and immunohistochemical staining of B7-H6 in normal cervical tissue (magnification, x200; scale bar, $100 \mu \mathrm{m}$ ). (B) B7-H6 differential expression was analyzed using H-score (CIN II compared with CINI, "P<0.05; CIN III compared with CINII, ${ }^{* * * *} \mathrm{P}<0.0001$; cervical cancer compared with CIN III, $\left.{ }^{*} \mathrm{P}<0.05\right)$. (C) H\&E and immunohistochemical staining of B7-H6 in CIN I, CIN II, CIN III and cervical cancer lesion tissues (magnification, x200; scale bar, $100 \mu \mathrm{m}$ ). B7-H6, B7 homolog 6; CIN, cervical intraepithelial neoplasia.

quadrants was counted for this analysis. The experiment was repeated three times.

Statistical analysis. The experimental data were processed by SPSS 19.0 statistical software (SPSS, Inc.). GraphPad Prism 8 (GraphPad Software, Inc.) was used to plot figures and verify the results. The data are shown as mean \pm standard deviation. One way ANOVA and Bonferonni's test were used for the comparison among multiple groups. Independent unpaired samples t test was used for comparison between two groups. $\mathrm{P}<0.05$ was considered to indicate a statistically significant difference.

\section{Results}

B7-H6 expression is associated with cervical cancer grade. B7-H6 expression levels were analyzed in normal cervical, CIN I, CIN II, CIN III and cervical cancer tissues using immunohistochemistry. The results revealed positive B7-H6 expression to differing intensities in CIN lesions and cervical cancer tissues. In further detail, no cells were found to express B7-H6 in normal cervical tissues (Fig. 1A), whereas in CIN I, CIN II, CIN III and cervical cancer tissues, the H-score of B7-H6 expression increased alongside the advancement in disease stage (Fig. 1B and C). Notably, the H-score of B7-H6 expression in CIN II tissues $(0.53 \pm 4.33)$ was significantly upregulated compared with that in CIN I tissues $(0.086 \pm 6.19)$; the H-score of B7-H6 expression in CIN III tissues (1.85 \pm 5.21$)$ was significantly upregulated compared with in CIN II tissues; and the $\mathrm{H}$-score of B7-H6 expression in cervical cancer tissues $(2.34 \pm 2.93)$ was significantly upregulated compared with in CIN III tissues.

Knockdown of B7-H6 expression in HeLa cells. The efficiency of infection of the HeLa cells with three groups of recombinant lentiviral vectors harboring B7-H6 shRNA was confirmed by analysis of GFP expression using fluorescence microscopy (Fig. 2A). Using RT-qPCR and western blotting analyses, the transfection of shRNA-1 (RT-qPCR, 0.22 \pm 0.02 ; western blotting, 28,538 $\pm 5,872$ ) or shRNA-2 (RT-qPCR, $0.11 \pm 0.01$; western blotting, 15,917 $\pm 3,750$ ) into HeLa cells significantly downregulated the expression levels of B7-H6 compared with HeLa cells transfected with shRNA-NC (RT-qPCR, 1.00 \pm 0.09 ; western blotting, 83,808 $\pm 5,282$; Fig. 2B and C).

Effect of B7-H6 on HeLa cell biological functions. The proliferation rate of HeLa cells following knockdown of B7-H6 expression was analyzed using CCK-8 and colony formation assays. The results of the CCK- 8 assay revealed that the viability of cells transfected with shRNA-1 (3 days, 
A
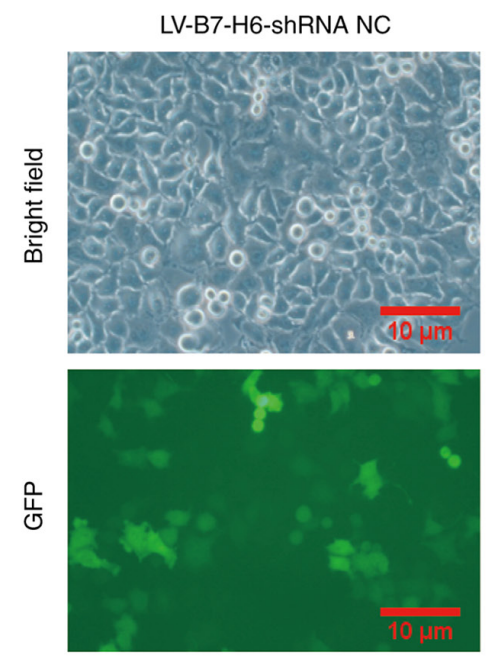

LV-B7-H6-ShRNA 1
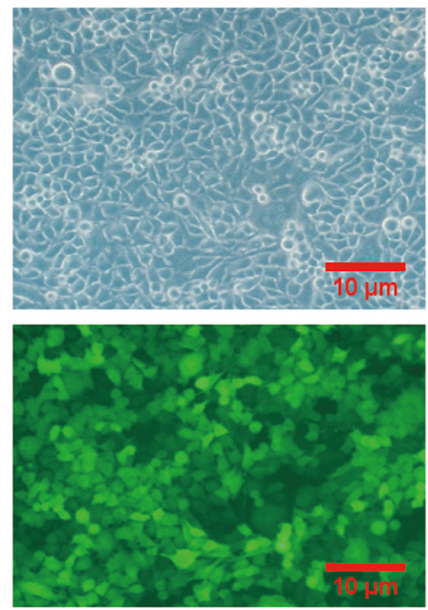

LV-B7-H6-shRNA 2
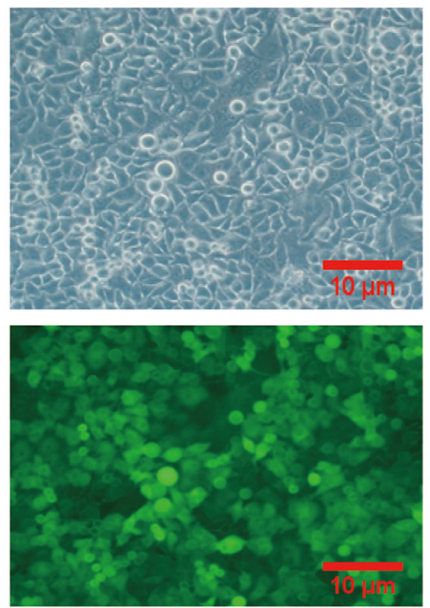

B

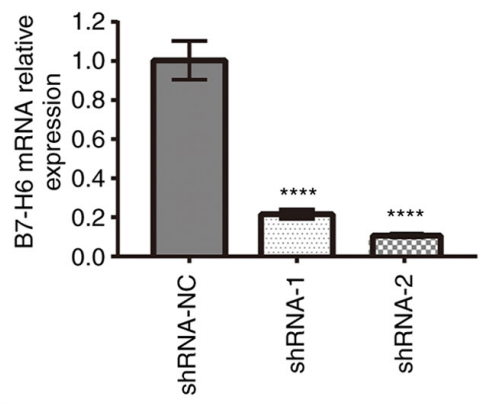

C

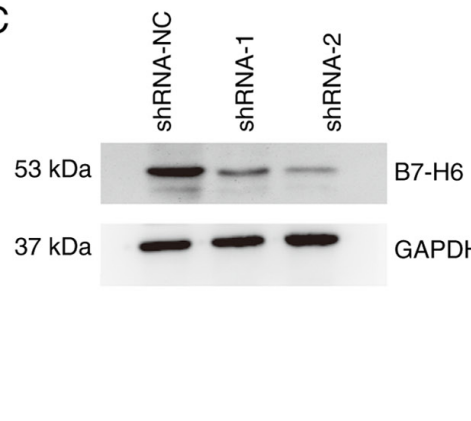

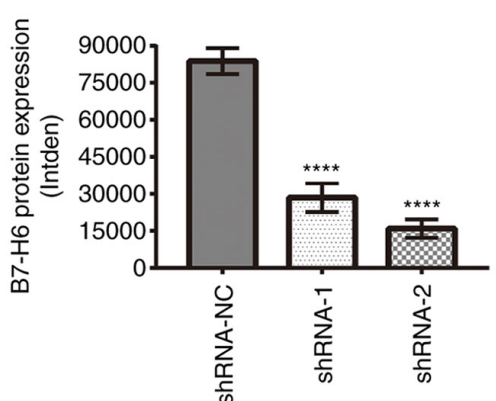

Figure 2. Knockdown of B7-H6 expression in HeLa cells. (A) Confirmation of the efficiency of infection of the HeLa cells with three groups of recombinant LV vector harboring B7-H6 shRNA by analysis of GFP expression using fluorescence microscopy (scale bar, 100 $\mu \mathrm{m}$ ). (B) Compared with shRNA-NC, the B7-H6 mRNA level decreased in the shRNA-1 and shRNA-2 groups, as shown by reverse transcription-quantitative PCR analysis ( $\left.{ }^{* * * *} \mathrm{P}<0.0001\right)$. (C) Compared with shRNA-NC, B7-H6 expression decreased in the shRNA-1 and shRNA-2 groups, as shown using western blotting (**** P<0.0001). B7-H6, B7 homolog 6; shRNA, short hairpin RNA; NC, negative control; LV, lentivirus; GFP, green fluorescent protein; intden, integrated density.

$1.40 \pm 0.08 ; 4$ days, $2.48 \pm 0.14$ ) or shRNA-2 (3 days, $0.96 \pm 0.11$; 4 days, $1.55 \pm 0.24)$ was significantly decreased compared with the cells transfected with shRNA-NC (3 days, 1.70 \pm 0.28 ; 4 days, 3.36 \pm 0.16 ; Fig. 3A). The results of the colony formation assay also demonstrated that, compared with the cells transfected with shRNA-NC $(89 \pm 0.91 \%)$, the colony formation rate of cells transfected with shRNA-1 (37 $\pm 1.25 \%)$ or shRNA-2 $(15 \pm 1.69 \%)$ was decreased following 1 week of culture (Fig. 3B).

Subsequently, wound healing and Transwell invasion assays were performed to determine the role of B7-H6 in HeLa cell migration and invasion. The wound healing assay results demonstrated that the cell-free area in plates containing cells transfected with shRNA-1 $(48 \mathrm{~h}$, $1.40 \pm 0.10 ; 72 \mathrm{~h}, 1.15 \pm 0.15)$ or shRNA-2 (48 h, 1.77 \pm 0.15 ; $72 \mathrm{~h}, 1.70 \pm 0.10)$ was significantly wider compared with cells transfected with shRNA-NC $(48 \mathrm{~h}, 1.07 \pm 0.15 ; 72 \mathrm{~h}$, $0.90 \pm 0.10$ ) (Fig. 3C). The results of the Transwell invasion assay revealed that the invasive ability of cells transfected with shRNA-1 $(73.33 \pm 7.37)$ or shRNA-2 $(57.33 \pm 3.48)$ was decreased compared with cells transfected with shRNA-NC

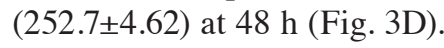

Effect of B7-H6 knockdown on HeLa cell apoptosis and cell cycle distribution. Flow cytometric analysis demonstrated that, compared with cells transfected with shRNA-NC $(3.91 \pm 4.22 \%)$, an increased proportion of cells transfected with shRNA-1 $(26.15 \pm 3.99 \%)$ or shRNA-2 $(33.32 \pm 7.24 \%)$ underwent late cell apoptosis (Fig. 4A). The results also demonstrated that the transfection with shRNA-2 increased the percentage of $\mathrm{G} 1$ cells $(46.78 \pm 1.99 \%)$ and decreased the percentage of $\mathrm{G} 2 / \mathrm{M}$ cells $(18.83 \pm 0.40 \%)$ compared with cells transfected with shRNA-NC (G1, 32.63 $\pm 2.84 \%$; G2/M, $31.80 \pm 2.69 \%$ ) (Fig. 4B).

\section{Discussion}

B7-H6, also known as NK cell toxicity receptor 3 ligand 1, belongs to the B7 immunoglobulin superfamily and, in recent years, has been discovered to act as a co-stimulator through bioinformatics analysis and mass spectrometry. The specific expression of B7-H6 in tumor cells has been shown to promote tumorigenesis (16) and to be associated with clinicopathological parameters $(19,20)$. The B7-H6 gene encodes a 454-amino acid type I transmembrane protein, with a predicted molecular mass of $51 \mathrm{kDa}$. Similar to all known B7 family members, B7-H6 comprises two immunoglobulin domains with adjacent phase 1 introns in the extracellular region (21). Although B7-H6 is expressed at very low levels, if at all, in normal tissues, its expression was found to be 


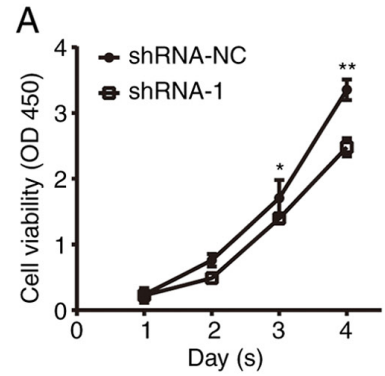

C
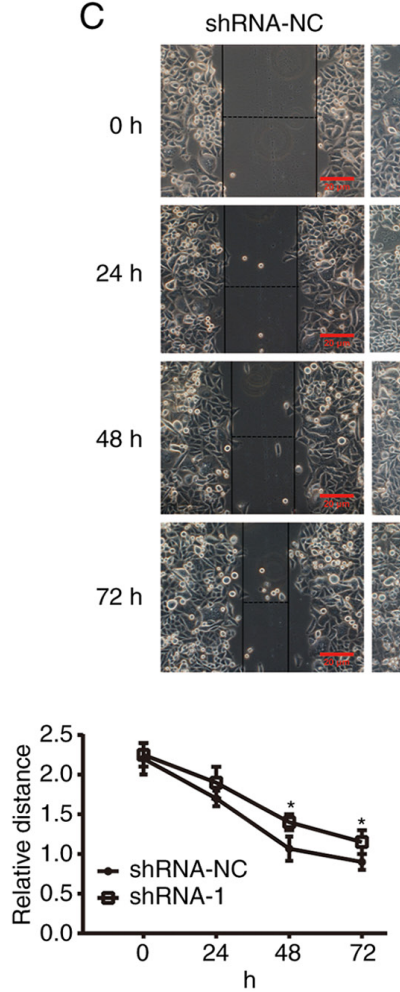

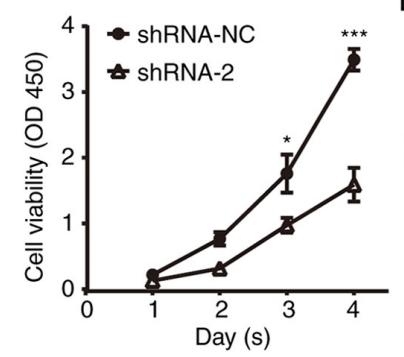

shRNA-1

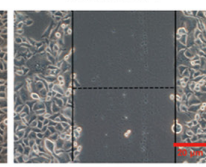

shRNA-2
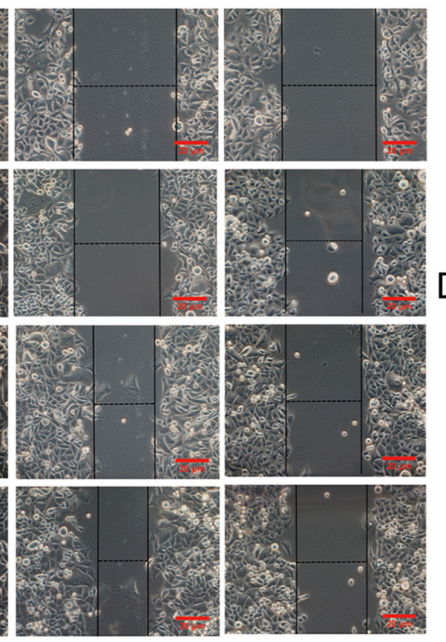

D

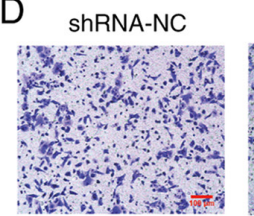

ShRNA-1
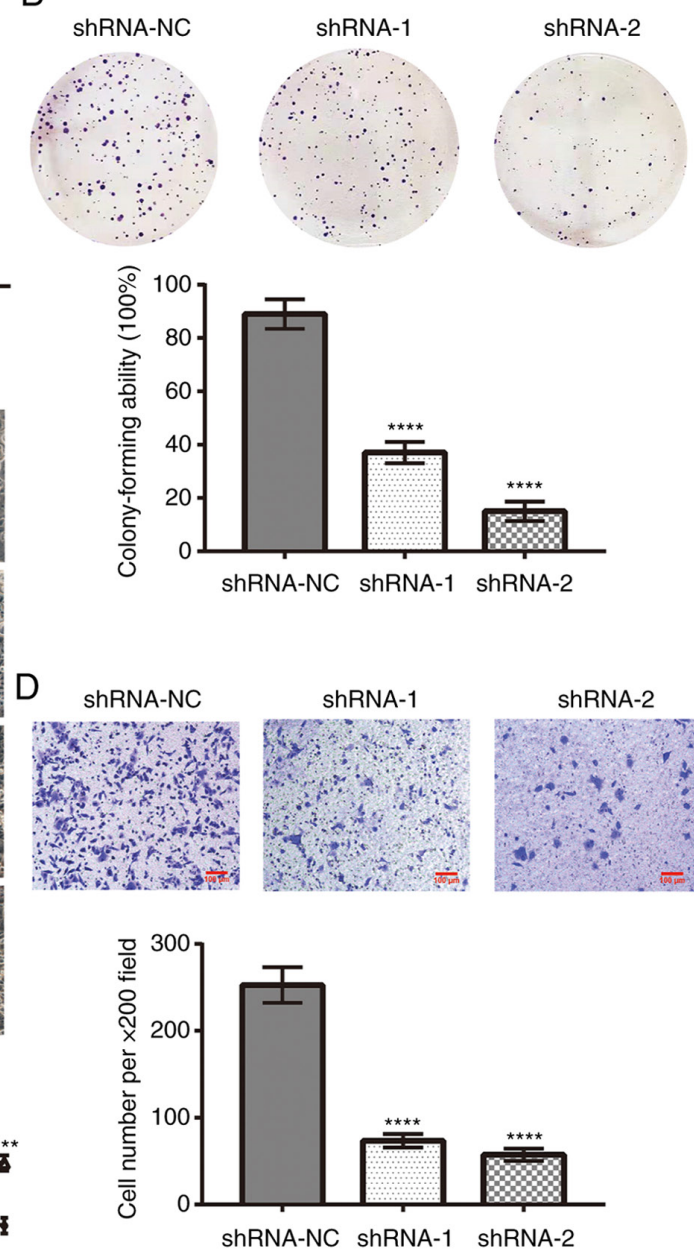

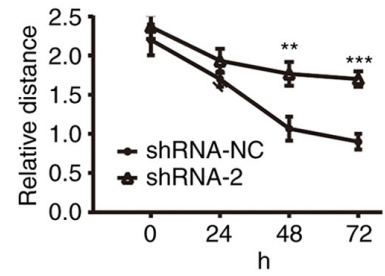

Figure 3. Effect of B7-H6 on HeLa cell biological functions. (A) B7-H6 knockdown by shRNA-1 and shRNA-2 inhibited cell viability, as shown by the Cell Counting Kit- 8 assay (shRNA-1 vs. shRNA-NC: 3 days, ${ }^{*} \mathrm{P}<0.05 ; 4$ days, ${ }^{* *} \mathrm{P}<0.01$; and shRNA-2 vs. shRNA-NC: 3 days, ${ }^{*} \mathrm{P}<0.05 ; 4$ days, ${ }^{* * *} \mathrm{P}<0.001$ ). (B) Colony formation assay demonstrated that B7-H6 knockdown inhibited the colony-forming ability of shRNA-1 and shRNA-2 cells (compared with shRNA-NC, ${ }^{* * * *} \mathrm{P}<0.0001$ ). (C) Wound healing assay demonstrated that the migration ability of HeLa cell was significantly weakened following B7-H6 knockdown (shRNA-1 vs. shRNA-NC at 48 and $72 \mathrm{~h},{ }^{*} \mathrm{P}<0.05$; shRNA-2 vs. shRNA-NC at $48 \mathrm{~h},{ }^{* * *} \mathrm{P}<0.01$; at $\left.72 \mathrm{~h},{ }^{* * * *} \mathrm{P}<0.001\right)$ (scale bar, $20 \mu \mathrm{m}$ ). (D) Transwell invasion assay demonstrated that the number of crystal violet-stained HeLa cells was decreased following B7-H6 knockdown (compared with shRNA-NC, ${ }^{* * * * *} \mathrm{P}<0.0001$ ) (scale bar, $100 \mu \mathrm{m}$ ). B7-H6, B7 homolog 6; shRNA, short hairpin RNA; NC, negative control.

upregulated in several types of cancer, including lymphoma, leukemia, hepatocellular carcinoma, gastric cancer, brain cancer, ovarian cancer and breast cancer (19,22-27). However, to the best of our knowledge, the clinical significance and role of B7-H6 in cervical cancer remain unclear.

The present study used immunohistochemistry analysis to demonstrate that the expression levels of B7-H6 were gradually upregulated as precancerous lesions developed into cervical cancer. These findings are consistent with previous findings in esophageal squamous cell carcinoma (16), breast cancer (17) and oral squamous cell carcinoma (19). B7-H6 was not only reported to be associated with the progression of these malignant tumors, but it was also closely associated with the clinicopathological parameters of the tumors and patient prognosis. Li et al (20) reported that the high expression of B7-H6 in gastric cancer tissues was significantly associated with overall survival. These aforementioned findings combined with the results of the present study suggested that the transcription of B7-H6 may be activated in cervical precancerous lesions and thereby serve an important role in the development of cervical cancer. B7-H6 expression may also be associated with clinicopathological parameters and prognosis in cervical cancer.

In the present study, subsequent cell experiments demonstrated that the expression levels of B7-H6 were successfully knocked down in HeLa cells using shRNA. CCK-8 and colony formation assays demonstrated that the proliferative ability of HeLa cells following B7-H6 knockdown was reduced. In addition, the migratory and invasive abilities of HeLa cells following B7-H6 knockdown were also significantly decreased, as determined using wound healing and Transwell invasion assays, respectively. Flow cytometry analysis also demonstrated that B7-H6 knockdown in HeLa cells promoted late apoptosis and cell cycle arrest at the G1 phase. These 

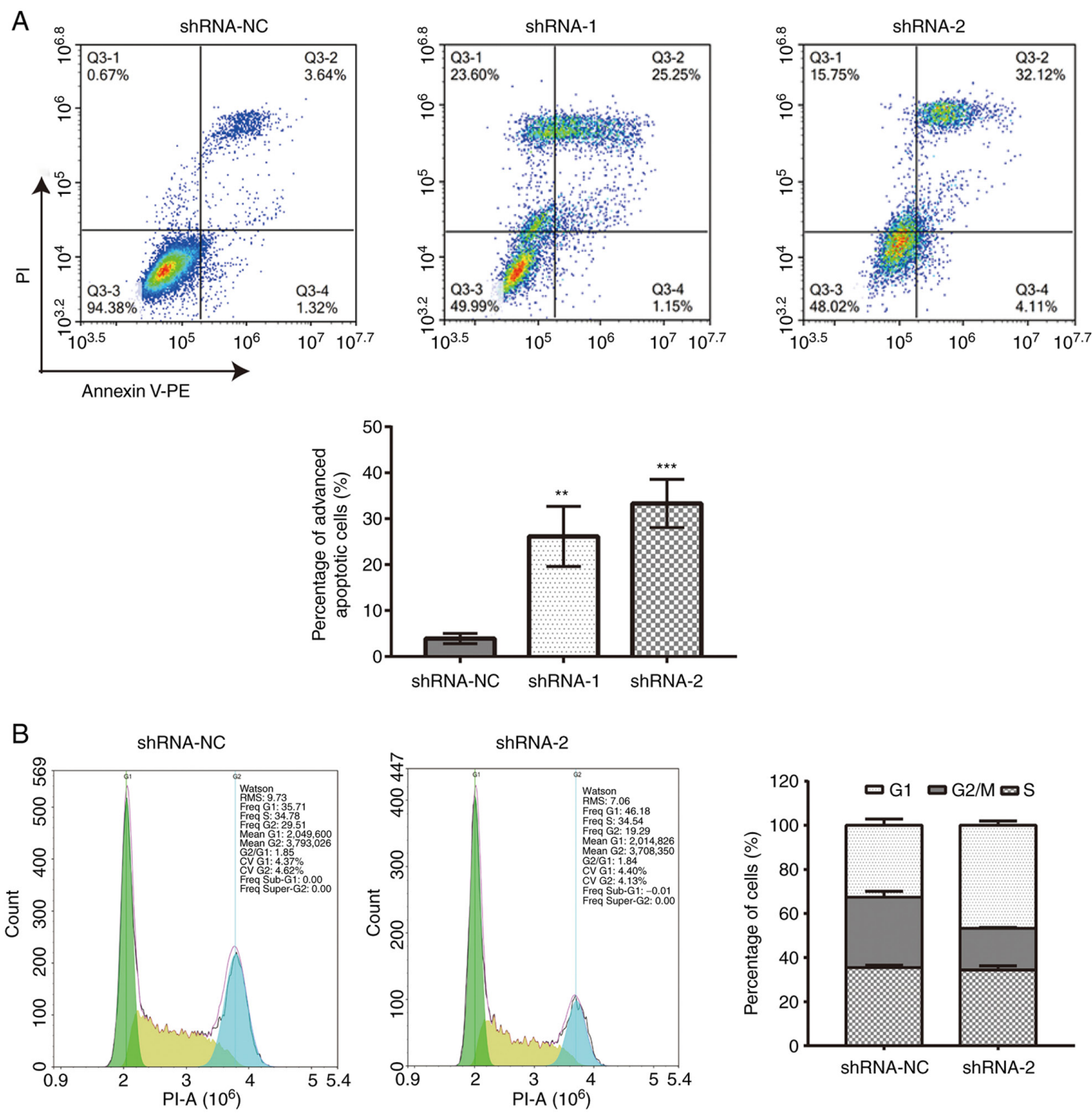

Figure 4. Effect of B7-H6 knockdown on HeLa cell apoptosis and cell cycle distribution. (A) Flow cytometric analysis demonstrated that the ratio of late apoptotic cells was significantly increased following B7-H6 knockdown (compared with shRNA-NC, shRNA-1, ${ }^{* *} \mathrm{P}<0.01$; and shRNA-2, $\left.{ }^{* * *} \mathrm{P}<0.001\right)$. (B) shRNA-2 cells displayed an increased percentage of G1 phase cells and a decreased percentage in the G2/M phase cells compared with the shRNA-NC (both P<0.0001). B7-H6, B7 homolog 6; shRNA, short hairpin RNA; NC, negative control.

findings were consistent with the results of Chen et al (22), which reported that the gene silencing of B7-H6 in the HepG2 and SMMC-7721 cell lines using shRNA significantly inhibited cell proliferation, migration and invasion, and induced cell apoptosis and cell cycle arrest by downregulating the expression levels of c-Myc, c-Fos and cyclin D1. Similarly, Che et al (27) reported that the proliferative and invasive abilities of glioma cell lines, including CRT, U251, SHG-44, SF-295, TG-905 and U373, were decreased following B7-H6 knockdown. In addition, the study also found that the expression levels of proteins associated with apoptosis, migration and invasion, including E-cadherin, Bcl-2, vimentin, N-cadherin, matrix metalloproteinase (MMP)-2, MMP-9 and survivin, were altered. Li et al (28) also suggested that B7-H6 may exert anti-apoptotic effects by upregulating the expression levels of MMP-9 and activating the STAT3 signaling pathway. In addition, in B cell lymphoma, B7-H6 gene silencing inhibited tumor cell colony formation, growth, invasion and migration, and induced cell apoptosis (29). These aforementioned studies and the results of the present study indicated that B7-H6 may promote cervical cancer tumorigenesis by regulating cell proliferation, cell cycle progression and apoptosis.

In conclusion, the results of the present study suggested that B7-H6 may be an oncogene and represent a novel tumor biomarker and potential target for cervical cancer treatment. Therefore, further investigations into the role of B7-H6 may provide novel insights into the treatment of cervical cancer. However, there were certain limitations to the present study. First, the possible mechanisms promoting B7-H6 transcription and the signaling pathway through which B7-H6 may regulate the proliferation, migration and apoptosis of cervical cancer cells remain unclear. Second, the mechanism of action of B7-H6 should be investigated in additional cervical cancer cell lines, and in vivo animal models should be used in future studies to verify the effect of B7-H6 in cervical cancer. Finally, further studies are required to determine the association between B7-H6 and the clinicopathological parameters and prognosis of cervical cancer. 


\section{Acknowledgements}

The authors would like to thank Dr Xue Du, Department of Gynecology, The General Hospital of Tianjin Medical University, Tianjin, China for providing the HeLa cell line.

\section{Funding}

The present study was supported by the Youth Fund of the Second Hospital of Tianjin Medical University, the Second Hospital of Tianjin Medical University (grant no. 2018ydey16) and The Natural Science Foundation of Tianjin, Tianjin Municipal Science and Technology Beaureau (grant no. 18JCYBJC28000).

\section{Availability of data and materials}

The datasets used and analysed during the present study are available from the corresponding author on reasonable request.

\section{Authors' contributions}

RG: Project development, data collection, data analysis, manuscript writing. GL: Project development. CL: Experimental technical support. XL: Data collection, conduction of experiments, data analysis. YX, WY and FW: Data collection. All the authors have read and approved the final manuscript. Authentication of the raw data: CL and XL.

\section{Ethics approval and consent to participate}

The present study was approved by the the Ethics Committee of the Second Hospital of Tianjin Medical University, and it is registered under the approval no. KY2019K023. All patients or their family members provided written informed consent for their tissues to be used for research purposes prior to participation.

\section{Patient consent for publication}

Not applicable.

\section{Competing interests}

The authors declare that they have no competing interests.

\section{References}

1. Stelzle D, Tanaka LF, Lee KK, Ibrahim Khalil A, Baussano I, Shah ASV, McAllister DA, Gottlieb SL, Klug SJ, et al: Estimates of the global burden of cervical cancer associated with HIV. Lancet Glob Health 2: e161-e169, 2020. Erratum in: Lancet Glob Health 2: e119, 2021.

2. Abu Rustum NR, Yashar CM, Bradley K, Campos SM, Chon HS, Chu C, Clinton L, Cohn D, Crispens MA, et al: NCCN Guidelines Version 1.2021 Cervical Cancer. J Natl Compr Canc Netw 6: 660-666, 2020.

3. Chitsike L and Duerksen-Hughes P: The potential of immune checkpoint blockade in cervical cancer: Can combinatorial regimens maximize response? A review of the literature. Curr Treat Options Oncol 21: 95, 2020.

4. Ni L and Dong C: New B7 family checkpoints in human cancers. Mol Cancer Ther 16: 1203-1211, 2017.
5. Hu Y,Zeng T, Xiao Z, Hu Q, Li Y, Tan X, Yue H, Wang W, Tan H and Zou J: Immunological role and underlying mechanisms of B7-H6 in tumorigenesis. Clin Chim Acta 502: 191-198, 2020.

6. Li Y, Wang Q and Mariuzza RA: Structure of the human activating natural cytotoxicity receptor NKp30 bound to its tumor cell ligand B7-H6. J Exp Med 208: 703-714, 2011.

7. Barrow AD, Martin CJ and Colonna M: The Natural cytotoxicity receptors in health and disease. Front Immunol 10: 909-913, 2019.

8. Joyce MG, Tran P, Zhuravleva MA, Jaw J, Colonna M and Sun PD: Crystal structure of human natural cytotoxicity receptor NKp30 and identification of its ligand binding site. Proc Natl Acad Sci USA 108: 6223-6228, 2011.

9. Fiegler N, Textor S, Arnold A, Rölle A, Oehme I, Breuhahn K, Moldenhauer $G$, Witzens-Harig $M$ and Cerwenka $A$ : Downregulation of the activating NKp30 ligand B7-H6 by HDAC inhibitors impairs tumor cell recognition by NK cells. Blood 122: 684-693, 2013.

10. Pesce S, Tabellini G, Cantoni C, Patrizi O, Coltrini D, Rampinelli F, Matta J, Vivier E, Moretta A, Parolini S, et al: B7-H6-mediated downregulation of NKp30 in NK cells contributes to ovarian carcinoma immune escape. OncoImmunology 4: e1001224, 2015.

11. Schlecker E, Fiegler N, Arnold A, Altevogt P, Rose-John S, Moldenhauer G, Sucker A, Paschen A, von Strandmann EP, Textor S, et al: Metalloprotease-mediated tumor cell shedding of B7-H6, the ligand of the natural killer cell-activating receptor NKp30. Cancer Res 74: 3429-3440, 2014.

12. Phillips M, Romeo F, Bitsaktsis C and Sabatino D: B7H6-derived peptides trigger TNF- $\alpha$-dependent immunostimulatory activity of lymphocytic NK92-MI cells. Biopolymers 106: 658-672, 2016.

13. Cao G, Wang J, Zheng X, Wei H, Tian Z and Sun R: Tumor therapeutics work as stress inducers to enhance tumor sensitivity to natural killer (NK) cell cytolysis by up-regulating NKp30 ligand B7-H6. J Biol Chem 290: 29964-29973, 2015.

14. Textor S, Bossler F, Henrich KO, Gartlgruber M, Pollmann J, Fiegler N, Arnold A, Westermann F, Waldburger N, Breuhahn K, et al: The proto-oncogene Myc drives expression of the NK cell-activating NKp30 ligand B7-H6 in tumor cells. Oncoimmunology 5: e1116674, 2016.

15. Zhou H, Dong J, Guo L, Wang X, Wang K, Cai X and Yang S: The prognostic value of B7-H6 in esophageal squamous cell carcinoma. Sci Rep 9: 18122-18130, 2019.

16. Sun J, Tao H, Li X, Wang L, Yang J, Wu P, Zhang Y and Guo Y: Clinical significance of novel costimulatory molecule B7-H6 in human breast cancer. Oncol Lett 14: 2405-2409, 2017.

17. Wang J, Jin X, Liu J, Zhao K, Xu H, Wen J, Jiang L, Zeng X, Li J and Chen Q: The prognostic value of B7-H6 protein expression in human oral squamous cell carcinoma. J Oral Pathol Med 46: 766-772, 2017.

18. Waxman AG, Chelmow D, Darragh TM, Lawson $\mathrm{H}$ and Moscicki AB: Revised terminology for cervical histopathology and its implications for management of high-grade squamous intraepithelial lesions of the cervix. Obstet Gynecol 120: 1465-1471, 2012.

19. Livak KJ and Schmittgen TD: Analysis of relative gene expression data using real-time quantitative PCR and the 2(-Delta Delta C(T)) Method. Methods 25: 402-408, 2001.

20. Li D, Xiang S, Shen J, Xiao M, Zhao Y, Wu X, Du F, Ji H, Li M, Zhao Q, et al: Comprehensive understanding of B7 family in gastric cancer: Expression profile, association with clinicopathological parameters and downstream targets. Int J Biol Sci 16: 568-582, 2020.

21. Chen Y, Mo J, Jia X and He Y: The B7 Family Member B7-H6: A new bane of tumor. Pathol Oncol Res 24: 717-721, 2018.

22. Chen L, Feng J, Xu B, Zhou Y, Zheng X, Wu C and Jiang J: B7-H6 expression in human hepatocellular carcinoma and its clinical significance [corrected]. Cancer Cell Int 18: 126-136, 2018. Erratum in: Cancer Cell Int 18: 134, 2018.

23. Jiang T, Wu W, Zhang H, Zhang X, Zhang D, Wang Q, Huang L, Wang Y and Hang C: High expression of B7-H6 in human glioma tissues promotes tumor progression. Oncotarget 8: 37435-37447, 2017.

24. Wu MR, Zhang T, Gacerez AT, Coupet TA, DeMars LR and Sentman CL: B7H6-specific bispecific T cell engagers lead to tumor elimination and host antitumor immunity. J Immunol 194: 5305-5311, 2015.

25. Chen XJ, Shen J, Zhang GB and Chen WC: B7-H6 protein expression has no prognostic significance in human gastric carcinoma. Pathol Oncol Res 20: 203-207, 2014. 
26. Xu Z, Shen J, Wang MH, Yi T, Yu Y, Zhu Y, Chen B, Chen J, $\mathrm{Li} \mathrm{L}, \mathrm{Li} \mathrm{M}$, et al: Comprehensive molecular profiling of the B7 family of immune-regulatory ligands in breast cancer. OncoImmunology 5: e1207841, 2016.

27. Che F, Xie X, Wang L, Su Q, Jia F, Ye Y, Zang L, Wang J, Li H, Quan Y, et al: B7-H6 expression is induced by lipopolysaccharide and facilitates cancer invasion and metastasis in human gliomas. Int Immunopharmacol 59: 318-327, 2018.

28. Li YM, Liu ZY, Li ZC, Wang JC, Yu JM, Yang HJ, Chen ZN and Tang J: Alterations of the immunologic co-stimulator B7 and TNFR families correlate with hepatocellular carcinoma prognosis and metastasis by inactivating STAT3. Int J Mol Sci 20: 156-174, 2019.
29. Wu F, Wang J and Ke X: Knockdown of B7-H6 inhibits tumor progression and enhances chemosensitivity in B-cell non-Hodgkin lymphoma. Int J Oncol 48: 1561-1570, 2016.

This work is licensed under a Creative Commons

Attribution-NonCommercial-NoDerivatives 4.0 International (CC BY-NC-ND 4.0) License. 